\title{
All transverse and TT tensors in flat spaces of any dimension
}

\section{J. Tafel $^{1}{ }^{1}$}

Received: 10 November 2017 / Accepted: 19 February 2018 / Published online: 26 February 2018 (C) The Author(s) 2018. This article is an open access publication

\begin{abstract}
We present general formulas for transverse and transverse-traceless (TT) symmetric tensors in flat spaces. TT tensors in conformally flat spaces can be obtained by means of a conformal transformation.
\end{abstract}

Keywords Transverse tensors · TT tensors $\cdot$ Conformal method

\section{Introduction}

A symmetric tensor $T^{i j}$ is called transverse if it satisfies

$$
\nabla_{i} T^{i j}=0
$$

where $\nabla_{i}$ denotes the covariant derivative with respect to metric $g_{i j}$ of a $D$-dimensional space. Condition (1) occurs in general relativity as an analog of the conservation law for energy and momentum, as the harmonic coordinate condition or as the momentum constraint in the initial data problem for the vacuum Einstein equations. In the last case one often assumes that, additionally,

$$
g_{i j} T^{i j}=0 .
$$

If (1) and (2) are satisfied then the tensor $T^{i j}$ is called transverse-traceless (TT). TT tensors were introduced by Arnowitt, Deser and Misner in 1959 [1].

$\otimes$ J. Tafel

tafel@fuw.edu.pl

1 Institute of Theoretical Physics, University of Warsaw, Warsaw, Poland 
Equation (1) is particularly simple if $\nabla_{i}$ is the covariant derivative corresponding to a flat connection. Even then its solutions are interesting, especially if (2) is satisfied, mainly because they can be used to construct initial vacuum data by means of the Lichnerowicz-York method [2,3]. This method is based on the invariance of Eqs. (1)(2) under the transformation

$$
g_{i j}^{\prime}=\psi^{\frac{4}{D-2}} g_{i j}, \quad T_{i j}^{\prime}=\psi^{-2} T_{i j}
$$

with a nonvanishing function $\psi$. Examples of conformally flat data obtained in this way can be found in classical papers by Misner [4], Brill and Lindquist [5], Bowen and York [6] and Brandt and Brügmann [7].

In this article we present general solution of equations

$$
T_{, j}^{i j}=0,
$$

with or without condition (2) with constant metric $g_{i j}$. They are expressed in terms of potentials which undergo gauge transformations. From them all TT tensors in conformally flat spaces can be obtained by means of transformation (3).

For obvious reasons we assume $D \geq 2$. All results are based on the Poincare lemma about local exactness of closed forms. For our purposes it is convenient to use the following form of this lemma

$$
\alpha_{, i_{p}}^{I i_{1} . . i_{p}}=0 \Rightarrow \alpha^{I i_{1} . . i_{p}}=\beta_{, i_{p+1}}^{I i_{1} . . i_{p+1}}
$$

where tensors $\alpha$ and $\beta$ are completely antisymmetric in indices $i_{1}, i_{2}, \ldots$ and $I$ denotes a set of additional indices. Integer $p$ satisfies $1 \leq p \leq D-1$.

\section{Solutions of $T^{i j}{ }_{j}=0$}

Proposition 1 All solutions of Eq. (4) have the form

$$
T^{i j}=R_{, k p}^{i k j p},
$$

where $R^{i k j p}$ is a tensor possessing all algebraic symmetries of the Riemann tensor.

Proof Let us apply the Poincare lemma (5) with $p=1$ and $I=i$ to Eq. (4). Hence, there exists tensor $S^{i j k}$ such that

$$
T^{i j}=S_{, k}^{i j k}, \quad S^{i k j}=-S^{i j k} .
$$

Condition $T^{i j}=T^{j i}$ yields

$$
S_{, k}^{[i j] k}=0 .
$$


Taking into account (5) with $I=[i j]$ we obtain potentials $V^{i j k p}$ such that

$$
S^{[i j] k}=V_{, p}^{i j k p}
$$

and

$$
V^{i j k p}=-V^{j i k p}=-V^{i j p k}
$$

A combination of Eq. (9) for different permutations of indices $i j k$ leads to

$$
S^{i j k}=\left(V^{i j k p}-V^{i k j p}-V^{j k i p}\right)_{, p} .
$$

Substituting (11) to (7) yields

$$
T^{i j}=-\left(V^{i k j p}+V^{j p i k}\right)_{, k p}
$$

Let $R^{i k j p}$ be defined by

$$
R^{i k j p}=-V^{i k j p}-V^{j p i k}+2 V^{[i k j p]}
$$

where the square bracket denotes antisymmetrization over all indices inside the bracket. Then (12) takes the form (6). Tensor $R^{i j k p}$ has algebraic symmetries of the Riemann tensor. It is antisymmetric in the first and second pair of indices, symmetric with respect to interchange of these pairs and $R^{[i j k p]}=0$ (hence also $R^{i[j k p]}=0$ ).

For $D=2$ one has

$$
R^{i k j p}=\frac{1}{2} R \epsilon^{i k} \epsilon^{j p}
$$

where $R$ is arbitrary function and $\epsilon^{i j}$ is the standard completely antisymmetric tensor. It follows from (6) that

$$
T^{i j}=\epsilon^{i k} \epsilon^{j p} R_{, k p}
$$

For $D \geq 3$ tensor $R^{i j k p}$ contains more components than number of degrees of freedom admitted by Eq. (4). Arbitrariness in choice of $R^{i j k p}$ describes the following proposition.

Proposition 2 For $D=2$ function $R$ is given up to the translation $R \longrightarrow R+R^{\prime}$, where

$$
R^{\prime}=a_{i} x^{i}+b, \quad a_{i}, b=\text { const } .
$$


For $D \geq 3$ tensor $R^{i k j p}$ is given up to the translation by

$$
R^{\prime i k j p}=\left(\frac{1}{2} \xi^{i k j p r}+\frac{1}{2} \xi^{j p i k r}-\xi^{[i k j p r]}\right)_{, r}, \quad \xi^{i k j p r}=\xi^{[i k][j p r]}
$$

Proof For $D=2$ an addition to $R$ does not change $T^{i j}$ if all its second derivatives vanish. Thus, it has to be linear in all coordinates.

In order to show that for $D \geq 3$ all solutions of the equation

$$
R_{, k p}^{\prime i k j p}=0
$$

with symmetries of the Riemann tensor, have the form (16) let us write (17) as

$$
\left(R_{, k}^{i k j p}\right)_{, p}=0
$$

From the Poincare lemma (5) with $I=i$ one obtains

$$
R_{, k}^{i k j p}=\frac{1}{2} V_{, k}^{i j p k}
$$

where tensor $V^{i j p k}$ is antisymmetric in the last 3 indices, $V^{i j p k}=V^{i[j p k]}$. Thanks to $R^{\prime[i j k] p}=0$ an antisymmetrization of (19) over the indices $i j$ leads to

$$
\left(R^{\prime i j p k}+V^{[i j] p k}\right)_{, k}=0
$$

Using again (5), now with $I=[i j]$, we obtain

$$
R^{\prime i j p k}+V^{[i j] p k}=\xi_{, r}^{i j p k r},
$$

where $\xi^{i p j k r}=\xi^{[i p][j k r]}$. Antisymmetrization of (21) over $i j p$ yields

$$
V^{[i j p] k}=\xi_{, r}^{[i j p] k r}
$$

Taking a suitable combination of Eq. (22) with different permutations of indices allows to express tensor $V$ in the form

$$
V^{i j p k}=\left(3 \xi^{[j p k] i r}+4 \xi^{[i j p k] r}\right)_{, r}
$$

Substituting (23) to (21) leads to (16).

In dimension $D=2$ gauge transformations (15) can be used to remove a constant term and terms linear in coordinates in an expansion of $R$ around a fixed point. In higher dimensions tensor $R^{i k j p}$ can be decomposed into a traceless part $C^{i k j p}$, corresponding 
to the Weyl tensor in general relativity, and the rest which is defined by an analog of the Ricci tensor $R_{k p}=R_{k i p}^{i}$

$$
R_{j p}^{i k}=C_{j p}^{i k}+4 a \delta_{[j}^{[i} R_{p]}^{k]}-2 b R \delta_{[j}^{[i} \delta_{p]}^{k]} .
$$

Here $R=R_{i}^{i}$ and

$$
a=\frac{1}{D-2}, \quad b=\frac{1}{(D-1)(D-2)} .
$$

Tensor $C^{i k j p}$ vanishes identically for $D=3$. For $D \geq 4$ we can try to achieve this condition by means of the gauge transformation (16).

Proposition 3 In dimension $D=3$ every solution and in $D \geq 4$ every analytic solution of (4) has the form

$$
T_{i j}=a \Delta R_{i j}-2 a R_{(i, j) k}^{k}+b R_{, i j}+\left(a R_{, k p}^{k p}-b \Delta R\right) g_{i j},
$$

where $R_{i j}$ is an arbitrary symmetric tensor undergoing the gauge transformation

$$
R_{i j} \longrightarrow R_{i j}+\xi_{(i, j)}-\xi_{, k}^{k} g_{i j}
$$

with arbitrary functions $\xi_{i}$.

Proof Equation (26) follows if (24) with $C^{i k j p}=0$ is substituted to (6). In order to prove that for $D \geq 4$ condition $C^{i k j p}=0$ is available one could look for an appropriate gauge transformation of the form given by Proposition 2. In our opinion it is easier to prove solvability of (26) with respect to $R_{i j}$ if a transverse tensor $T^{i j}$ is given. Let us distinguish coordinate $x^{1}$, which together with $x^{a}, a=2, \ldots D$, composes a Cartesian system of coordinates. Concerning evolution of $R_{i j}$ with respect to $x^{1}$ Eq. (26) with indices 11 and $1 a$ are constraints since they do not contain second derivatives of $R_{i j}$ over $x^{1}$. It is easy to show that they are preserved in $x^{1}$ if they are satisfied at $x^{1}=0$ and remaining Eq. (26) are fulfilled. If functions $T_{i j}$ are analytic then the constraints at $x^{1}$ admit solutions and from the Cauchy-Kowalewska theorem we obtain analytic solutions $R_{i j}$ of all Eq. (26). This situation is similar to that in general relativity. Equations (26) are identical with the linearized Einstein equations if $R_{i j}-\frac{1}{D-1} R g_{i j}$ is identified with the first corrections to the constant metric $g_{i j}$. This analogy suggests the gauge transformations (27). It is easy to show that they preserve the rhs of (26). Counting number of components of $R_{i j}$ and $\xi_{i}$ we can be sure that transformations (27) are general up to functions of $D-1$ variables. In order to exclude such additional functions one should find all transformations (16) preserving $C^{i k j p}=0$ and investigate their efect on $R_{i j}$. It hasn't been done.

Remark If $g_{i j}$ has the Lorentzian signature then Eq. (26) for $R_{i j}$ are hyperbolic and the assumption about analyticity in Proposition 3 can be weakened. 


\section{TT tensors}

For $T^{i j}$ given by expression (6) the traceless condition (2) leads to the following equation for the "Ricci tensor" $R^{i j}=R_{i k j}^{k}$

$$
R_{, k p}^{k p}=0
$$

Equation (28) can be easily solved in terms of potentials.

Proposition 4 For $D=2$ TT tensors are given by (14), where $R$ satisfies

$$
\triangle R=0
$$

For $D \geq 3$ TT tensors are given by (6) and

$$
R^{p k}=S_{, r}^{(p k) r},
$$

where

$$
S^{p k r}=-S^{p r k}
$$

Proof For $D=2$ there is $R^{i j}=\frac{1}{2} R g^{i j}$, so (28) reduces to Eq. (29), which can be further integrated by means of holomorphic functions (for a definite signature of $g_{i j}$ ) or functions of null coordinates (for mixed signature).

For $D \geq 3$ there is no other restrictions on $R^{p k}$ except (28). Integrating (28) with use of (5) yields

$$
R_{, k}^{p k}=V_{, k}^{p k}, \quad V^{p k}=-V^{k p}
$$

and integrating (32) leads to

$$
R^{p k}=V^{p k}+S_{, r}^{p k r},
$$

where new potentials $S^{p k r}$ satisfy (31). Symmetrization of (33) over indices $p k$ yields (30).

Potentials $S^{i j k}$ are not uniquely defined. Their arbitrariness can be easily defined in the case of gauge $C^{i j k p}=0$.

Proposition 5 If (26) and (30) are satisfied then potentials $S^{i j k}$ undergo gauge transformations

$$
S^{i j k} \longrightarrow S^{i j k}-2 g^{i[j} \xi^{k]}+\chi_{, r}^{i j k r}+\eta^{i j k},
$$

where

$$
\chi^{i j k r}=\chi^{i[j k r]}, \quad \eta^{i j k}=\eta^{[i j k]} .
$$


Proof In order to prove (34) one should solve condition

$$
S_{, k}^{\prime(i j) k}=0
$$

for an addition to $S^{i j k}$ which does not influence $R^{i j}$ given by (30). From (36) and (5) it follows that

$$
S^{\prime(i j) k}=V_{, r}^{i j k r},
$$

where $V^{i j k r}=V^{(i j)[k r]}$. If we define $\chi^{i j k r}=\frac{3}{8} V^{i[j k r]}$ then $V^{i j k r}=4 \chi^{(i j) k r}$. Taking into account the identity

$$
S^{\prime i j k}=S^{\prime[i j k]}+\frac{2}{3} S^{\prime(i j) k}-\frac{2}{3} S^{\prime(i k) j}
$$

one obtains

$$
S^{\prime i j k}=\chi_{, r}^{i j k r}-\chi_{, r}^{[i j k] r}+S^{\prime[i j k]} .
$$

Denoting two last expressions in (39) by $\eta^{i j k}$ leads to transformation (34).

An application of transformation (34) with $\xi^{k}=-S_{i}{ }^{i k} /(D-1)$ and vanishing all other terms allows to obtain $S_{i}^{i k}=0$ and $R=0$. Then substituting (30) into (26) yields

$$
T_{i j}=a\left(\Delta S_{(i j), k}^{k}+S_{(i, j) k r}^{k r}\right)
$$

An appropriate choice of $\eta$ allows to get $S^{[i j k]}=0$. Further gauge conditions can be assumed thanks to free functions $\chi^{i j k r}$.

A description of TT tensors in dimension $D=3$ is much simpler than in $D \geq 4$. In this case Propositions 4 and 5 lead to the following result.

Proposition 6 In dimension $D=3$ every TT tensor is given by

$$
T^{i j}=\epsilon^{k l(i}\left(\triangle A_{k}^{j)}-A_{k p,}^{j) p}\right)_{, l},
$$

where $A_{i j}$ is a symmetric tensor undergoing the gauge transformations

$$
A_{i j} \longrightarrow A_{i j}+\chi_{(i, j)}+\eta g_{i j}
$$

with arbitrary functions $\chi_{i}$ and $\eta$.

Proof For $D=3$ one has $C^{i k j p}=0$ and

$$
R^{i k j p}=-\epsilon^{i k l} \epsilon^{j p s} G_{l s},
$$


where $G_{l s}=R_{l s}-\frac{1}{2} R g_{l s}$ corresponds to the Einstein tensor. Substituting (64) to (6) leads to the following simplified version of formula (26)

$$
T^{i j}=-\epsilon^{i k l} \epsilon^{j p s} G_{l s, k p} .
$$

Gauge transformation (27) is now the most general since

$$
\xi^{i k j p r}=-\xi_{l} \epsilon^{l i k} \epsilon^{j p r}
$$

should be substituted into (16). If $T_{i}^{i}=0$ then $R^{p k}$ is given by (30), where

$$
S^{p k r}=\epsilon^{k r l} A_{l}^{p} .
$$

Tensor $A$ can be arbitrary, but only its symmetric part appears in $G_{l s}$. Without loss of generality we can assume

$$
A^{l p}=A^{p l}
$$

hence the gauge condition $S_{i}{ }^{i k}=0$ is satisfied. Substituting (46) to (30) and using (44) leads to (41).

Tensor $A$ can be further simplified by means of (34). Since for $D=3$

$$
\chi^{i j k r}=\chi^{i} \epsilon^{j k r}, \quad \eta^{i j k}=\eta \epsilon^{i j k}
$$

condition $S_{i}{ }^{i k}=0$ is preserved if

$$
\xi^{k}=\frac{1}{2} \epsilon^{k i j} \chi_{[i, j]}
$$

Substituting (46), (48) and (49) to (34) yields (42).

Remark If $T^{\prime i j}$ is a transverse tensor then

$$
T^{i j}=\epsilon^{k l(i} T_{k, l}^{\prime j)}
$$

is a TT tensor. Case (41) corresponds to

$$
T^{\prime i j}=\triangle A^{i j}-2 A_{p,}^{(i) p}+A_{, p k}^{p k} g^{i j} .
$$

If $D \geq 4$ one can consider gauge conditions for a TT tensor other than $C^{i j k r}=0$. A natural candidate is condition $R^{i j}=0$.

Proposition 7 In dimension $D \geq 4$ every analytic TT tensor has the form

$$
T^{i j}=C_{, k p}^{i k j p}
$$

where $C^{i k j p}$ is a tensor with all algebraic properties of the Weyl tensor. 
Proof Formula (52) is true if transformations (16) allow to kill $R^{i j}$. Let us look for solutions of equations

$$
\xi_{, r}^{(k p) r}=-R^{k p}
$$

where

$$
\xi^{k p r}=\xi_{i}^{i k p r}
$$

Let $x^{1}$ and $x^{a}$ be coordinates introduced in the proof of Proposition 3. Equation (53) with $k=p=1$ is a constraint regarding evolution of $\xi^{k p r}$ with respect to $x^{1}$. The derivative of this constraint over $x^{1}$ leads to the new constraint

$$
\xi_{, a b}^{(a b) 1}=R_{, 1}^{11}+2 R_{, a}^{1 a} \text {. }
$$

Condition (55) is preserved if $T_{i}^{i}=0$ since then $R_{, k p}^{k p}=0$. Thus, if we assume (53) with $k=p=1$ and (55) on the surface $x^{1}=0$ then, in analytic case, we obtain solution of all Eq. (53). It remains to prove that for this solution there is a tensor $\xi^{i k j p r}$ which satisfies (54). It is the case since every such tensor admits decomposition

$$
\xi_{i k}^{j p r}=\hat{\xi}_{i k}^{j p r}+\alpha \delta_{[i}^{[j} \xi_{k]}^{p r]}-\beta \delta_{[i}^{[j} \delta_{k]}^{p} \xi^{r]},
$$

where $\hat{\xi}_{i k}^{j p r}$ is traceless and

$$
\alpha=\frac{6}{D-3}, \quad \beta=\frac{6}{(D-2)(D-3)}, \quad \xi^{r}=\xi_{k}^{k r} .
$$

It follows from (56) that tensors $\hat{\xi}^{i k j p r}$ and $\xi^{k p r}$ can be defined independently.

If $R^{i j}=0$ then gauge transformations (16) are restricted by $\xi_{, r}^{(k p) r}=0$. It follows from (36)-(39) that solution of this condition is

$$
\xi^{i j k}=\chi_{, r}^{i j k r}+\eta^{i j k}
$$

where tensors $\chi$ and $\eta$ satisfy (35). Transformations (16) with condition (58) can be further used to simplify formula (52).

\section{Discussion}

All calculations were performed in Cartesian coordinates $x^{i}$. If we replace partial derivatives with respect to $x^{i}$ by covariant derivatives $\nabla_{k}$ then we can pass to other systems of coordinates. In particular formula (6) then takes the form

$$
T^{i j}=\nabla_{k} \nabla_{p} R^{i k j p}
$$


For a nonflat metric expression (59) fails to satisfy (1) because of nonvanishing commutators of covariant derivatives.

Given solutions of Eqs. (1) and (2) in a flat space we can obtain all TT tensors in conformally flat spaces by means of transformation (3). The author is not able to express in general these solutions in terms of covariant derivatives of potentials with no use of the conformal factor $\psi$. The only exception is the case of spaces of constant curvature (space forms corresponding to flat space, sphere or hyperbolic space). Then

$$
R_{k p}^{i j}=\lambda\left(\delta_{k}^{i} \delta_{p}^{j}-\delta_{k}^{j} \delta_{p}^{i}\right), \lambda=\text { const }
$$

and Eq. (1) is still satisfied if formula (59) is replaced by

$$
T^{i j}=-\nabla_{k} \nabla_{p} R^{k(i j) p}+\frac{1}{2} \lambda R^{i j} .
$$

In order to satisfy $T_{i}^{i}=0$ it is sufficient, but may be not necessary, to assume the covariant version of (30)

$$
R^{p k}=\nabla_{r} S^{(p k) r}
$$

together with

$$
S^{i j k}=-S^{i k j}, \quad S_{i}^{i k}=0 .
$$

We expect that for $D \geq 4$ the gauge condition $C^{i j k l}=0$ is available for transverse tensors and condition $R^{i j}=0$ for TT tensors.

Let us assume that space is flat but a TT tensor is invariant under a symmetry of metric. Such solutions can be obtained by means of invariant tensor potentials appearing in Propositions 1-7. Such description is not necessarily optimal as it follows from the paper of Conboye and Ó Murchadha [8] and Conboye [9] in dimension $D=3$. Their expressions for TT tensors contain only two arbitrary functions and at most their second derivatives, not third as in (41).

For a general metric $g$ we are not able to write down solutions of (4), with or without $H=0$, in terms of potentials. In the case of $D=3$ and axially symmetric fields Eq. (4) can be completely solved in generic case (see Propositions 2.1 and 2.2 in [10]), but then condition $H=0$ becomes a differential equation.

In this paper we focused on mathematical description of transverse and transversetraceless tensors. Concerning physical applications we are mainly interested in using results of Sect. 3 in order to construct initial data within the conformal approach to the vacuum Einstein equations. Following results of Maxwell [11] one can include in these data marginally trapped surfaces which are expected to develop into black hole horizons. Such configurations would generalize those of Bowen and York [6]. They are often considered as nonphysical since the Kerr metric does not admit any conformally flat section. However, the conformal flatness property will be spoiled during the time evolution. It would be interesting to see if the Kerr like black holes will be created in agreement with the cosmic censor hypothesis. We are going to consider a related 
but simpler problem: using our data and Maxwell's approach we will test the Penrose inequality involving the surface area of an initial trapped surface and the total mass of the initial surface.

Another possible application of our approach would be a construction of cosmological initial data describing perturbations of the standard cosmological models (note that surfaces of constant time in the Friedmann-Robertson-Walker spacetimes are always conformally flat).

An example of condition (4) in general relativity is provided by the de Donder condition $\left(\sqrt{|g|} g^{\mu \nu}\right)_{, v}=0$. It is specially useful in the linearized gravity in vacuum since, together with the Einstein vacuum equations, it leads to equations

$$
\begin{aligned}
h^{i j}{ }_{, j} & =0, \quad h_{i}^{i}=0 \\
\square h_{i j} & =0
\end{aligned}
$$

(where $\square=\eta^{\mu \nu} \partial_{\mu} \partial_{\nu}$ ) for corrections $h_{i j}$ to the space components of the Minkowski metric $\eta_{\mu \nu}$. In the standard approach solutions of these equations are obtained as linear superpositions of harmonic waves. Using our results we can go further by taking $h_{i j}$ in the form (41) with matrix $A$ satisfying the unconstrained wave equation (65). Note that functions $A_{i j}$ can be found e.g. by means of separation of variables what is not possible for $h_{i j}$ because of conditions (64).

We hope that other physical applications appear with time.

Open Access This article is distributed under the terms of the Creative Commons Attribution 4.0 International License (http://creativecommons.org/licenses/by/4.0/), which permits unrestricted use, distribution, and reproduction in any medium, provided you give appropriate credit to the original author(s) and the source, provide a link to the Creative Commons license, and indicate if changes were made.

\section{References}

1. Arnowitt, R., Deser, S., Misner, C.W.: The dynamics of general relativity. In: Witten, L. (ed.) Gravitation: An Introduction to Current Research, pp. 227-265. Wiley, Hoboken (1962)

2. Lichnerowicz, A.: J. Math. Pures Appl. 23, 39-63 (1944)

3. York Jr., J.W.: Gravitational degrees of freedom and the initial-value problem. Phys. Rev. Lett. 26, 1656 (1971)

4. Misner, C.W.: The method of images in geometrostatics. Ann. Phys. 24, 102-117 (1963)

5. Brill, D.R., Lindquist, R.W.: Interaction energy in geometromechanics. Phys. Rev. 131, 471-476 (1963)

6. Bowen, J.M., York Jr., J.W.: Time-asymmetric initial data for black holes and black hole collisions. Phys. Rev. D 21, 2047-2055 (1980)

7. Brandt, S.R., Brügmann, B.: A simple construction of initial data for multiple black holes. Phys. Rev. Lett. 78, 3606-3609 (1997)

8. Conboye, R., Ó Murchadha, N.: Potentials for transverse trace-free tensors. Class. Quantum Gravity 31, 085019 (2014)

9. Conboye, R.: Coordinate independent expression for transverse trace-free tensors. Class. Quantum Gravity 33, 015008 (2016)

10. Tafel, J., Jóźwikowski, M.: New solutions of initial conditions in general relativity. Class. Quantum Gravity 31, 115001 (2014)

11. Maxwell, D.: Solutions of the Einstein constraint equations with apparent horizon boundaries Commun. Math. Phys. 253, 561-583 (2005) 